\title{
Problems associated with clinical trials of Ayurvedic medicines
}

\author{
Ashish K Sharma, ${ }^{* 1}$ Rajesh Kumar, ${ }^{2}$ Anurag Mishra, ${ }^{3}$ Rajiv Gupta ${ }^{3}$ \\ ${ }^{1}$ Adarsh Vijendra Institute of Pharmaceutical Sciences, Gangoh, Saharanpur-247431, Uttar Pradesh, India \\ ${ }^{2}$ Faculty of Pharmacy, R. P. Educational Trust Group of Institutions, Karnal-132001, Haryana, India \\ ${ }^{3}$ Department of Pharmacognosy, Faculty of Pharmacy, Babu Banarasi Das National Institute of Technology \& \\ Management, Dr. Akhilesh Das Nagar, Lucknow-227 105 (U.P.) India.
}

RESUMO: Problemas associados com ensaios clínicos de medicamentos Ayurvédicos.

\begin{abstract}
Herbal drugs have been used since ancient times as medicines for the treatment of various diseases. Especially in countries like India many of herbal drugs and formulations are used in different practices of treatment like Ayurveda, Siddha and Unani. It is estimated that about $25 \%$ of all modern medicines are directly or indirectly derived from plants sources. The contribution of developing countries in global herbal business is very poor due to lack of quality control and standardization measures. There is lack of common standards and appropriate methods for evaluating Traditional Medicine to ensure the safety, efficacy and quality control. This indicates the importance and necessity to develop a standard operational procedure for the standardization of herbal drugs and formulations. Benchmarking the evaluation protocols including both quality control and quality assurance of herbal drugs would play a major role in providing highly reliable and effective herbals drugs and to attract international trade, thus generating revenue. The article highlights various problems being faced by developing countries and suggests a unique approach for the preparation of SOP/guidelines for the standardization of all herbal based formulations, also there is a need for systematic clinical trials of traditional plant based medicines to enhance global acceptance.
\end{abstract}

Keywords: Clinical trials, good manufacturing practice, placebo, World Health Organization.

\section{INTRODUCTION}

The origin of practice of medicine developed gradually and separately in ancient Egypt, ancient China, ancient India, ancient Greece, Persia and elsewhere. This slow and progressive development led to the birth of the modern pharmaceutical industry. In spite of the great advances observed in modern synthetic medicine in recent decades, plants still make an important contribution to health care. According to the World Health Organization (WHO) estimates, because of poverty and lack of access to modern medicine, about $65-80 \%$ of the world's population which lives in developing countries depends essentially on plants for primary health care. Currently, the major pharmaceutical companies have demonstrated renewed interest in investigating higher plants as sources for new lead structures along with development of standardized phytotherapeutic agents with proven efficacy, safety, and quality. Herbal medicinal preparations are normally very popular in developing countries because of being economical, backed with a long history of traditional use and also in some developed countries such as Germany, France, Italy and United States where appropriate guidelines for registration of such medicines exist (Brevoort, 1995; Wagner, 1999; Calixto, 2000).

\section{REASONS FOR THE INCREASED WORLDWIDE INTEREST OF HERBAL MEDICINES}

During the past decades, public interest in natural therapies, namely herbal medicine, has increased dramatically not only in developing countries but also in industrialized countries. This has renewed the international trade in herbal medicine enormously and has attracted most of the pharmaceutical companies, including the multinationals (Shiapush, 1998).

Several important factors have contributed to the growth of this worldwide phytotherapeutic market, among which some of the following may be mentioned below (McLennan et al., 1996):

- Preference of consumers for natural therapies;

- Concern regarding undesirable side effects of modern medicines and the belief that herbal drugs are free from side effects, since millions of people all over the world have been using herbal medicines for thousands of years;

- Some conventional therapies, where treatment paradigm is quite slow, psychologically patient finds solace in herbals;

- Tendency towards self-medication;

- Improvement in quality, proof of efficacy and 
safety of herbal medicines;

- High cost of synthetic medicines;

- People who experience dissatisfaction or limited success with the outcome of orthodox medicine, to complementary medicine most commonly have conditions associated with chronic pain (back and neck injuries, arthritis and rheumatism) or illnesses such as cancer whereas orthodox medicine deals primarily with treating the symptoms (Tapsell et al., 2006);

- The popularity of complementary medicine has also been supported by the research efforts into their safety and effectiveness, although a lack of scientifically valid research into complementary medicine has always been a significant barrier to orthodox medicine in accepting these health care approaches (Robbers \& Tyler, 1998).

\section{EVALUATION OF THE EFFICACY AND SAFETY OF HERBAL MEDICINES WITH CONTROLLED CLINICAL TRIALS}

In 2004 the U.S. National Center for Complementary and Alternative Medicine of the National Institutes of Health began funding to support clinical trials exploring the effectiveness of herbal medicine. The universal standard for pharmaceutical testing is repeated, large-scale, randomized double-blind tests. To recoup the considerable costs of testing to the regulatory standards, the substances are patented by pharmaceutical companies and sold at a substantial profit.

Many herbs have shown positive results in in vivo animal model or small-scale clinical tests but many studies on herbal treatments have also given negative results. The quality of the trials on herbal remedies is of high quality and many trials of herbal treatments have exhibited poor quality, with many trials lacking an intention to treat analysis or a comment on whether trial protocol was successful. The few randomized, double-blind tests that attracted attention in mainstream medical publications are often questioned on the basis of methodological grounds or interpretation in terms of reproducible results (Eisenberg et al., 1993; Robbers \& Tyler, 1999; Pittler et al., 2000).

Dosage is another a long pending issue for herbal treatments, while most conventional medicines are aggressively tested to determine the most effective and safest dosages (especially in relation to parameters like body weight, drug interactions etc.), there are few established dosage standards for various herbal treatments available in the market (Linde et al., 2001; Srinivasan, 2005).

Several factors might contribute to of such issues $\&$ discrepancies, for example:

- Lack of standardization and quality control of the herbal drugs used in clinical trials;

- Use of different dosages of herbal medicines;

- Inadequate randomization in most studies, and patients batch not properly selected;
- Numbers of patients in most trials are insufficient for the attainment of statistical significance;

- Difficulty in establishing appropriate placebos because of the taste and aroma etc;

- Wide variations in the duration of treatments using herbal medicines.

\section{MAJOR PROBLEMS WITH CLINICAL TRIALS OF AYURVEDIC MEDICINES}

- Ayurvedic HMPs (Herbal Medicinal Products) are marketed as dietary supplements, they are regulated under the Dietary Supplement Health and Education Act (DSHEA), which does not require proof of safety or efficacy and hence bypasses the stringent quality tests of Drugs and Cosmetics Act 1940. Recently with use of Ayurvedic HMPs Lead toxicity has been reported, which might result in conditions like status epilepticus, fatal infant encephalopathy, congenital paralysis, sensoryneural deafness, and foetal developmental delay. Since 1978, at least 65 cases of heavy metal intoxication associated with Ayurvedic HMPs in adults and children have been reported in the United States and abroad (Eisenburg et al., 1998; Robert et al., 2004).

- Conducting clinical trials on traditional/herbal medicines is quite challenging due to lot of reasons, for instance, it is very difficult, impracticable or sometimes impossible to have active and control groups with identical color, smell and taste. Also, the use of placebo involves similar difficulties as the herbal study drug may exhibit its strong aroma, a specific distinguished taste and hence these cannot be exactly imitated while manufacturing a placebo. The comparative placebo use in evaluation of herbal drugs also raises ethical issues as it is unethical to assign unaware patients a placebo instead of using an available therapy effective in treating their condition (Rothman \& Michels, 1994).

- Quite often the problems are multiplied when working with formulations containing more than one plant extracts. Special attention must also be paid on standardization of the compounds using bioactive markers.

- There are difficulties in estimation of "active" molecules in body fluids in the pharmacokinetic studies in phase I of drug development especially in Ayurvedic formulation (Bhattaram et al., 2002).

- Storage conditions can also alter the bioavailability of herbal medicines, which may lead to loss of activity, fungal or bacterial contamination resulting into batch to batch variation, are the bane of large clinical trials with medicinal plants (Thatte, 2005).

- Importance of Good Manufacturing Practices are generally not given due consideration while manufacturing Ayurvedic products hence, while working with marketed formulation, it is necessary to keep a check on adulteration and substitution. 
- Selection of dosage regimen may also pose problems. Dose must be calculated on the basis that whether crude drug or extract is used, and the dose of the extract has to be calculated on the basis of extractive value (Thatte, 2005).

- The system of herbal medicine advocates the choice of herbs, compounding or mixtures, dosage and duration so that it suits the particular psychosomatic constitution (Prakriti) of the patient. It is well known in Ayurveda that the "Prakriti" determines the effectiveness of particular herbal medicines which influences response to drugs and also a major factor to consider as an inclusion/ exclusion criterion in clinical studies. This approach, since ancient times, helps in enhancing the therapeutic effect as well as reduces the unwanted effects of the drug. Therefore, administration of a study drug to a subject population of various constitutions may not yield uniform outcomes (Dahanukar \& Thatte, 1997).

- Many a times the medicine system advocates other procedures along with oral therapy which again may or may not be standardized. Also, there is no standard single benchmark formulation, especially if a single drug therapy is concerned for various therapeutic areas, as they are present in modern medical science.

- Another reason is that the crude raw herb material has number of variations as per geographical locations, climatic conditions, environmental hazards, harvesting methods, collection protocols, etc which makes it a difficult task to standardize the end product for a reproducible quality (Bauer \& Tittel, 1996).

- Other reasons include the fact that integrated approach of many herbal systems does not differentiate the disease from the patient. This approach creates difficulties for the inclusion and exclusion criteria in clinical trial.

- Also, Ayurveda proposes different interventions at different stages of disease in the same patient providing another difficult to control variable in a clinical trial.

- Diet component may also affect the treatment outcome in clinical trial.

- As most herbal medicines used in traditional systems are used in the humans without any toxicity studies, their embryotoxic, foetotoxic, and carcinogenic effects are likely to remain unrecognized in traditional practice. Recently the embryotoxic effects of 'Pippalyadi vati' (traditional contraceptives consisting of Embelia ribes, Piper longum and Borax) have been reported (Chaudhary et al., 2001, Chan, 2003).

- Other reasons include the fact that integrated approach of many herbal systems does not differentiate the disease from the patient. This approach creates difficulties for the inclusion and exclusion criteria in clinical trial.

- With Ayurvedic medicines, it is difficult to plan double-blind randomized clinical trials. For clinical evidence of safety and efficacy the placebo-controlled, double blind randomized trial may be considered as gold standard, even for herbal medicine. However, randomized clinical trials on herbal medicine may be quite difficult to conduct because it needs large volunteer groups, is of long duration and requires expensive healthcare services. Moreover, one major problem that exists is that the funds for such trials, is usually not readily available (Shukla et al.,, 1991; Armstrong \& Ernst, 1999).

- One key issue in planning clinical trials is that some herbs have been reported to be poisonous (Moore \& Adler, 2000).

- The problem with the use of herbal medicines is the fact that it is not always clear whether interactions exist between herbal medicine and other medicinal products or substances like alcohol, caffeine, tobacco or nicotine or with diet. Some of the common herb-drug interactions includes: a) Bleeding- when warfarin is combined with Ginkgo biloba, garlic, dong quai or danshen; b) Serotonin syndrome- when St. Johns' wort is combined with serotonin- reuptake inhibitors; and c) Induction of mania in depressed patients - when antidepressants are mixed with Panax ginseng (Fugh-Berman, 2000).

- The issues associated with safety and quality of herbal medicines include toxic herbs, contamination with heavy metals, microbial organisms, and other contaminants (such as pesticides) as well as deliberate combination (adulteration) with pharmaceutical products.

- Another problem with research on alternative medicine is the fact that complementary health practitioners often question the suitability of randomized clinical trials in relation to complementary medicine because of the holistic approach (i.e. treating mind, body and soul at the same time) that is required and the fact that the alternative medicine is normally tailored specifically for each individual. Practitioners suggest it is inappropriate to test only one treatment in isolation and to give it to all participants in the same manner and amount, as occurs in randomized clinical trials (Kristofferson, 1996).

As a function of such difficulties, few herbal drugs have been studied adequately but well-controlled doubleblind clinical trials to prove their safety and efficacy have been lacking. e.g. Although the clinical trials including the extract of Ginkgo biloba (used for the treatment of CNS and cardiovascular disorders) and Hypericum perforatum (St. John's wort, used as an antidepressant) (Ernst et al., 1998) have shown that these two herbal drugs are quite safe and devoid of serious side effects, their clinical efficacy still requires well-controlled randomized double-blind studies. Other botanical drugs such as Panax ginseng (ginseng) herbs used as a tonic, Tanacetun parthenium (feverfew) used to treat migraine headache, Allium sativum (garlic) used to lower serum low-density protein, cholesterol level and some cardiovascular disturbances, Matricaria chamomilla (chamomile) recommended as a carminative, anti-inflammatory and antispasmodic, Silybium marianun (milk thistle) used for normalizing liver function including cirrhosis, Valeriana officinalis (valerian), used as a sedative and sleeping aid, Piper methysticum (Kava kava) used as 
an anxiolytic, Aesculus hippocastanum (horse chestnut) used for the treatment of chronic venous insufficiency, Cassia acutfolia (Senna) and Rhamnus purshiana (cascara sagrada) which are used as laxatives, Echinacea purpura (Echinacea) used as an anti-inflammatory and immunostimulant, Arnica montana (arnica) used to treat post-traumatic and postoperative conditions, and Serenoa repens (saw palmetto) used for the treatment of benign prostatic hyperplasia are all herbal medicines with the largest worldwide market, and although they have been evaluated in different clinical trials, additional wellcontrolled and appropriate randomized clinical trials are still needed in order to prove their efficacy (Bauer \& Tittel, 1996; Harrer et al. 1999; Armstrong \& Ernst, 1999).

\section{STATUS OF CLINICAL TRIALS OF AYURVEDIC MEDICINE}

Due to the fact that plants cannot be patented, very little research has been performed on plants as medicinal agents. In USA the process of demonstrating drug safety and efficacy of a new pharmaceutical takes approximately 15 years and costs an estimated $\$ 500$ million and only few research companies are willing to fully invest the time and money necessary to satisfy the FDA requirements. It is therefore quite evident that the regulatory requirements for proof of safety and efficacy generally make it uneconomical for the private industry to conduct costly clinical trials on herbal medicine. If, however, the regulatory requirements regarding efficacy can be relaxed, private companies might more easily pursue research into issues of safety and quality control of herbal medicine. Public funds might still however be required to confirm the validity of herbal remedies, because pharmaceutical companies would earn meager incentive to develop a herbal product that might displace a patented drug (Vaidya et al., 2003). It is therefore argued that more data generation is needed to assess the efficacy than to assess safety of herbal medicine and, for proof of efficacy, the clinical trial has become the gold standard to majority of population. In fact it is argued that unless a proper study has been conducted on human subjects, no pertinent conclusion on its efficacy and or safety can be drawn. Further, the infrastructure for alternative and herbal medicine research is largely nonexistent (Thatte et al., 1993;).

\section{HERBAL DRUG DEVELOPMENT}

In recent years, we have witnessed the increasing growth in popularity of over-the-counter (OTC) health foods, nutraceuticals and medicinal products from plants or other natural sources in developed and developing countries. These OTC products may be contaminated with excessive or banned pesticides, microbial contaminants, heavy metals and chemical toxins which may be related to the source of these herbal materials, if they are grown under contaminated environment or during careless collection of these plant materials (Fisher \& Ward, 1994). Some of these environment related factors can be controlled by implementing standard operating procedures (SOP) leading to Good Agricultural Practice (GAP), Good Laboratory Practice (GLP), Good Supply Practice (GSP) and Good Manufacturing Practice (GMP) for producing these medicinal products from herbal or natural sources. The public's belief that herbal and natural products are safer than synthetic medicines can only be ascertained by imposing regulatory standards on these products that should be manufactured using this Good Practices (Grunwald, 1995).

Herbal drug development includes various steps, starting from a passport data on raw materials, correct identification, pharmacognostic and chemical quality standardization, safety and preclinical pharmacology, clinical pharmacology and randomized, controlled clinical trials. Addressing standardization is vital and needs broader consideration. Ayurvedic medicine was in practice at times of limited access to technologically variable norms of standardization. Standardization of Ayurvedic botanicals and medicines is seriously required, although one cannot readily apply the typical modern pharmaceutical Pharmacopoeial standards. The concept of active markers in the process of standardization needs a flexible approach in favor of the complex nature of these herbal materials. Recently, many international authorities and agencies, including the World Health Organization, European Agency for the Evaluation of Medicinal Products and European Scientific Cooperation of Phytomedicine (ESCOP), US Agency for Health Care Policy and Research, European Pharmacopoeia Commission, Department of Indian System of Medicine has started developing new mechanisms to induce and regulate quality control and standardization of botanical medicine. For Ayurvedic medicine and other traditional medicines, newer and regularly updated guidelines of standardization are required. Preclinical studies on Ayurvedic medicines are of great importance for validating drug safety resulting from new procedures, or extractions are used during its preparation. The value of animal testing to establish safety and toxicity is not so critical if the botanicals are used in traditional forms. Suitable animal models help in understanding the mechanism of action or pharmacodynamics of medicines (Straus, 2002). However, for bringing more objectivity and also to confirm traditional claims, systematic clinical trials are not only necessary but are essential.

\section{CONCLUSION}

A uniform research policy in herbal medicines is need of the hour. The legal status of herbal ingredients varies from country to country. Use of herbals in low dose on the basis of experience only by a traditional practitioner does not assure safety, hence when these herbs are used 
in high doses and in concentrated form over a longer duration of period without medical guidance, may cause serious undesirable effects. Nevertheless, all the critical Pharmacopoeial tests such as dissolution time, microbial, pesticide and heavy metals contamination, etc. must be in accordance with global standards. It is important to ensure that all the Ayurvedic medicines manufactures are in accordance with current good manufacturing procedures for herbal products. Development of standardized herbal formulations is underway as an initiative of the Council for Scientific and Industrial Research (CSIR), and New Millennium Indian Technology Leadership Initiative (NMITLI) (Drews, 1995; Marcus \& Grollman, 2002). It will be in the interest of pharmaceutical companies, researchers and ultimately the global community to respect the traditions and build on their knowledge and experiential wisdom. Inadequate regulation and an increasing demand for better documentation of efficacy and safety of herbal remedies have countered this popularity. Under these circumstances, it has become imperative to examine some of the challenging issues that are being faced by clinical research trials with herbal medicines.

The pre-clinical and clinical trials for new ayurvedic drug formulations have been prescribed by the Indian Government Department of AYUSH (India) with the intention of providing appropriate evaluation methods to facilitate the development of regulation and registration in ayurveda and other traditional systems of medicines. Many a times the physician might refer to other nonconventional treatment methods along with oral therapy which again may or may not be standardized. Without the knowledge obtained from clinical trials, people using herbal medicine may be at risk for serious effects from taking the wrong dose, using the treatment in the wrong way or using it with other treatment with which it may interact. Furthermore, the results of randomized clinical trials on herbal medicine might go a long way to uncover new knowledge that will lead to better health for everyone.

Evidence of the efficacy of traditional herbal medicines may then be factual and not mere opinions. The goals of these suggestions are to facilitate the scientific evaluation and the eventual integration of traditional medicine into the national healthcare system, critically assisting an eventual rational use of traditional medicine through development of technical guidelines and international standards.

The conclusion drawn from this review is that presently developing countries lacked the necessary guidelines for clinical trials on Ayurvedic/natural/herbal medicines. If the WHO guidelines can be adopted in developing countries to approve clinical studies without the need for stringent safety data, clinical trials on Ayurvedic medicine can be encouraged to be at par with pharmaceutical products.

\section{REFERENCES}

Armstrong NC, Ernst E 1999. The treatment of eczema with Chinese herbs: a systematic review of randomized clinical trials. Brit J Clin Pharmacol 48: 262-264.

Bauer R, Tittel G 1996. Quality assessment of herbal preparations as a precondition of pharmacological and clinical studies. Phytomedicine 2: 193-198.

Bhattaram VA, Graefe U, Kohlert C, Veit M, Derendorf H 2002. Pharmacokinetics and bioavailability of herbal medicinal products. Phytomedicine 3: 1-33.

Brevoort P 1995. The U.S. botanical market: An overview. Herbal Gram 36: 49-59.

Calixto JB 2000. Efficacy, safety, quality control, marketing and regulatory guidelines for herbal medicines (phytotherapeutic agents). Braz J Med Biol Res 33: 179189.

Chaudhary Roy, Chandrashekharan M, Mishra S 2001. Embryotoxicity and teratogenicity studies of an ayurvedic contraceptives-pippalyadi vati. $J$ Ethnopharmacol 74:189-193.

Chan K 2003. Some aspects of toxic contaminants in herbal medicines. Chemosphere 52: 1361-1371

Dahanukar SA, Thatte UM 1997. Current status of ayurveda in phytomedicine. Phytomedicine 4: 359-368.

Drews J 1995. Intent and coincidence in pharmaceutical discovery. The impact of biotechnology. ArzneimittelForschung 45: 934-939

Eisenburg DM, Davis RB, Ettner SL, Appeal S, Wilkey S, Van Rompay M, Kessler RC 1998. Trends in alternative medicine use in the United States, 1990-1997, Results of a follow-up national survey. JAMA-J Am Med Assoc 280: $1569-1575$.

Eisenberg DM, Kessler RC, Foster C, Norlock FE, Calkins DR \& Delbanco TL 1993. Unconventional medicine in the United States. New Engl J Med 328: 246-252.

Ernst E, Rand JI, Stevinson C 1998. Complementary therapies for depression - An overview. Arch Gen Psychiat 55: 1026-1032.

Fisher P, Ward A 1994. Complementary medicine in Europe. Brit Med J 309: 107-111.

Fugh-Berman A 2000. Herb-drug interactions. Lancet 355:134138.

Grunwald J 1995. The European phytomedicines market: figures, trends, analysis. Herbal Gram 34: 60-65.

Harrer G, Schimidt U, Kuhn U, Biller A 1999. Comparison of equivalent between the St. John's wort extract LoHyp-57 and fluoxetine. Arzneimittel-Forschung 49: 289-296.

Kristofferson S 1996. Uptake of alternative medicine in Australia. Lancet 347: 569-573

Linde K, Jonas WB, Melchart D, Willich S 2001. The methodological quality of randomized controlled trials of homeopathy, herbal medicines and acupuncture. Int $J$ Epidemiol 30: 526-531.

Marcus D, Grollman A 2002. Botanical medicines - The need for new regulations. New Engl J Med 347: 2073-2076. 
MacLennan AH, Wilson DH, Taylor AW 1996. Prevalence and cost of alternative medicine in Australia. Lancet 347: 569-573.

Moore C, Adler R 2000. Herbal vitamins: lead toxicity and developmental delay. Am Acad Pediatrics 106: 600-602.

Pittler MH, Abbot NC, Harkness EF, Ernst E 2000. Location bias in controlled clinical trials of complementary/alternative therapies. Int J Epidemiol 53: 485-489.

Robbers JE, Tyler VE 1999. Tyler's Herbs of Choice: The Therapeutic Use of Phytomedicinals. The Haworth Herbal Press, Inc., New York. pp 124-158.

Rothman KJ, Michels KB 1994. The continuing unethical use of placebo controls. N Engl J Med 331: 394-398.

Robert BS, Stefanos K, Janet P, Michael JB, David ME, Roger BD, Russell SP 2004. Heavy Metal Content of Ayurvedic Herbal Medicine Products. JAMA 292:2868-73

Shiapush M 1998. Postmodern values, dissatisfaction with conventional medicine and popularity of alternative therapies. $J$ Sociol 34: 58-70

Srinivasan K 2005. Spices as influencers of body metabolism: an overview of three decades of research. Food Res Int 38: 77-86.

Shukla NK, Narang R, Nair NGK, Radhakrishna S, Satyavati G 1991. Multicentric randomized controlled clinical trial of Kshaarasootra (Ayurvedic medicated thread) in the management of fistula-in-ano. Indian J Med Res 94: 177185.

Straus S 2002. Herbal medicines - What's in the bottle? New Engl J Med 347: 1997-1998.

Tapsell LC, Hemphill I, Cobiac L, Patch CS, Sullivan DR, Fenech M, Roodenrys S, Keogh JB, Clifton PM, Williams PG, Fazio VA, Inge KE 2006. Health benefits of herbs and spices: the past, the present, the future. Med J Aust 185 (4 Suppl): S4-24.

Thatte UM, Rege NN, Phatak S, Dahanukar SA 1993. The flip side of Ayurveda. J Postgrad Med 39: 179-182.

Thatte UM 2005. Clinical resaerch with ayurvedic medicines. Pharma Times 37: 9-10.

Vaidya RA, Vaidya ADB, Patwardhan B, Tillu G, Rao Y 2003. Ayurvedic pharmacoepidemiology: a proposed new discipline. J Assoc Physicians India 51:528.

Wagner H 1999. Phytomedicine Research in Germany. Environ Health Perspect 107: 779-781. 